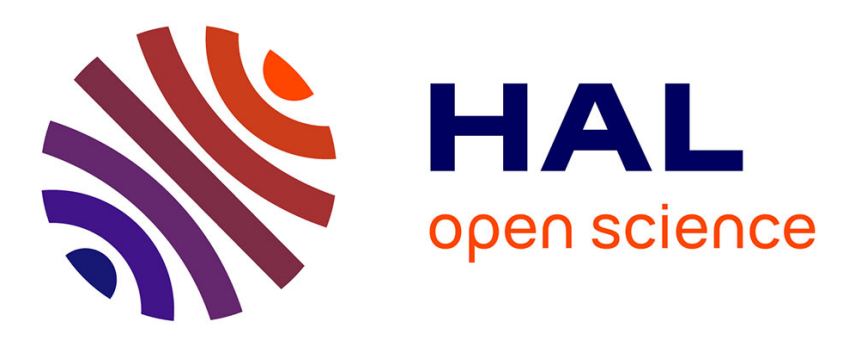

\title{
Phenotypic Characterization of a Leishmania donovani Cyclophilin 40 Null Mutant
}

\author{
Wai-Lok Yau, Ulrike Lambertz, Lucie Colineau, Pascale Pescher, Andrea \\ Macdonald, Dorothea Zander, Silke Retzlaff, Julia Eick, Joachim Clos, Neil E \\ Reiner, et al.
}

\section{To cite this version:}

Wai-Lok Yau, Ulrike Lambertz, Lucie Colineau, Pascale Pescher, Andrea Macdonald, et al.. Phenotypic Characterization of a Leishmania donovani Cyclophilin 40 Null Mutant. Journal of Eukaryotic Microbiology, 2016, 63 (6), pp.823-833. 10.1111/jeu.12329 . pasteur-01451083

\section{HAL Id: pasteur-01451083}

https://hal-pasteur.archives-ouvertes.fr/pasteur-01451083

Submitted on 28 Jan 2019

HAL is a multi-disciplinary open access archive for the deposit and dissemination of scientific research documents, whether they are published or not. The documents may come from teaching and research institutions in France or abroad, or from public or private research centers.
L'archive ouverte pluridisciplinaire HAL, est destinée au dépôt et à la diffusion de documents scientifiques de niveau recherche, publiés ou non, émanant des établissements d'enseignement et de recherche français ou étrangers, des laboratoires publics ou privés. 


\title{
Phenotypic Characterization of a Leishmania donovani Cyclophilin 40 Null Mutant
}

\author{
Wai-Lok Yau ${ }^{a, b, 1}$, Ulrike Lambertz ${ }^{c, 2}$, Lucie Colineau $^{c}$, Pascale Pescher ${ }^{a}$, Andrea MacDonald ${ }^{b}$, \\ Dorothea Zander ${ }^{\mathrm{b}}$, Silke Retzlaff ${ }^{\mathrm{d}, 3}$, Julia Eick ${ }^{\mathrm{b}}$, Neil E. Reiner ${ }^{\mathrm{c}}$, Joachim Clos $^{\mathrm{b}}$ \& Gerald F. Spätha \\ a Unité de Parasitologie Moléculaire et Signalisation, Institut Pasteur and Institut National de la Santé et de la Recherche Médicale INSERM \\ U1201, 25 rue du Dr Roux, F-75015 Paris, France \\ b Clos Group (Leishmanasis), Bernhard-Nocht-Institut für Tropenmedizin, Bernhard-Nocht-Street 74, D-20359 Hamburg, Germany \\ c Division of Infectious Diseases, Jack Bell Research Centre, University of British Columbia, 2660 Oak Street, Vancouver, British Columbia V6H \\ 3Z6, Canada \\ d Electron Microscopy Service, Bernhard-Nocht-Institut für Tropenmedizin, Bernhard-Nocht-Street 74, D-20359 Hamburg, Germany
}

\section{Keywords \\ Chaperone; exosomes; infection; null mutant analysis; proteomics; stress protein; trypanosomatid parasite; virulence. \\ Correspondence \\ G.F. Späth, Institut Pasteur, 25 rue du Dr Roux, 75015 Paris, France \\ Telephone number: +33140613858; \\ FAX number: +33 1456883 33; \\ e-mail: gerald.spaeth@pasteur.fr}

Received: 8 April 2016; revised 7 May 2016; accepted May 11, 2016.

doi:10.1111/jeu.12329

\section{ABSTRACT}

Protozoan parasites of the genus Leishmania adapt to their arthropod and vertebrate hosts through the development of defined life cycle stages. Stage differentiation is triggered by environmental stress factors and has been linked to parasite chaperone activities. Using a null mutant approach we previously revealed important, nonredundant functions of the cochaperone cyclophilin $\mathbf{4 0}$ in L. donovani-infected macrophages. Here, we characterized in more detail the virulence defect of cyp40-/- null mutants. In vitro viability assays, infection tests using macrophages, and mixed infection experiments ruled out a defect of cyp40-1- parasites in resistance to oxidative and hydrolytic stresses encountered inside the host cell phagolysosome. Investigation of the CyP40dependent proteome by quantitative 2D-DiGE analysis revealed up regulation of various stress proteins in the null mutant, presumably a response to compensate for the lack of CyP40. Applying transmission electron microscopy we showed accumulation of vesicular structures in the flagellar pocket of cyp40-1parasites that we related to a significant increase in exosome production, a phenomenon previously linked to the parasite stress response. Together these data suggest that cyp40-/- parasites experience important intrinsic homeostatic stress that likely abrogates parasite viability during intracellular infection.
EARLY-BRANCHING eukaryotes of the trypanosomatid family represent some of the most important human pathogens, including Trypanosoma cruzi, Trypanosoma brucei, and Leishmania spp. that cause South American Chagas Disease, African sleeping sickness and Leishmaniasis respectively (McCall and McKerrow 2014). These parasites are transmitted to humans by blood feeding insects and adapt to their arthropod or mammalian hosts through the development of defined life cycle stages. This adaptation is triggered by changes in the physiology of the host environment. Leishmania spp. for example grow extracellularly at neutral $\mathrm{pH}$ and moderate temperature as procyclic promastigotes inside the midguts of infected sand flies. In response to nutritional starvation after sand fly blood meal excretion, the parasites develop into growth arrested, highly virulent metacyclic promastigotes and following transmission, they differentiate into the diseasecausing, intracellular amastigote stage in response to $\mathrm{pH}$ and temperature shock inside the phago-lysosome of host macrophages (Zilberstein and Shapira 1994). Thus, parasite development and infectivity are fundamentally associated with the Leishmania stress response.

A first functional link between Leishmania spp. stage differentiation and the parasite response to environmental stress was established by pharmacological inhibition. Treatment of $L$. donovani promastigotes with the HSP9O inhibitors geldanamycin or radicicol resulted in the development of amastigote-like forms in the absence of $\mathrm{pH}$ and temperature shock, suggesting that this major chaperone is required to maintain the promastigote phenotype 
(Wiesgigl and Clos 2001). A similar phenotype has been observed using cyclosporin $A$, an inhibitor that targets members of the cyclophilin (CyP) protein family (Tedesco and Haragsim 2012), which are characterized by a peptidylprolyl isomerase (PPlase) activity implicated in protein folding and signal transduction (Wang and Heitman 2005). CsA treatment of $L$. donovani promastigotes caused cell cycle arrest and profound morphological changes, including loss of motility, retraction of the parasite flagella, and ovoid cell shape, reminiscent of developing amastigotes (Yau et al. 2010). While CsA treatment had no effect on promastigote viability, treatment of axenic amastigotes caused massive cell death, thus uncovering stage-specific functions in the regulation of parasite thermotolerance.

The evolutionary expansion of the Leishmania CyP protein family with 17 highly conserved members and the stage-specific toxicity of CsA suggest important roles of PPlases in trypanosomatid biology. Trypanosomatid CyPs have been implicated in a variety of cellular functions, ranging from protein disaggregation to subversion of host defense mechanisms or parasite drug resistance (Chakraborty et al. 2002; Kulkarni et al. 2013; Matrangolo et al. 2013; Moro et al. 1995; Mukherjee et al. 2013). Despite the importance of this protein family in parasite development and infectivity, only little is known about the unique functions of the individual members of this family in pathogenic trypanosomatids.

Using a null mutant approach, we previously investigated the functions of $L$. donovani CyP40. CyP40 is a bifunctional member of the CyP family that not only carries PPlase activity but also plays an important role as cochaperone, forming dynamic complexes with HSP90 in yeast and mammalian cells through a conserved tetratricopeptide repeat (TPR) domain (Hoffmann and Handschumacher 1995; Pratt et al. 2004). Ablation of this protein in L. donovani did not affect parasite viability in logarithmically growing culture but caused dramatic morphological defects in parasites entering stationary growth phase that could be linked to a disorganization of the parasite subpellicular tubulin network (Yau et al. 2014). Cyp40-/- parasites failed to establish intracellular infection despite expression of metacyclic markers, revealing important nonredundant functions for this cyclophilin in parasite virulence. Here, we combined macrophage infection assays with quantitative proteomics and structural analyses with the aim to investigate in more detail the mechanisms causing loss of intracellular survival in this mutant. Cyp40-/- parasites showed normal oxidant resistance in vitro and parasite survival was not rescued in macrophages that lacked antimicrobial activities or that were co-infected with wildtype (WT) parasites, pointing to an intrinsic defect as the cause of virulence attenuation. This view was further supported by our observations that cyp40-1- parasites compensated for the absence of this important cochaperone by increasing the abundance of various stress proteins, and showed a significantly enhanced production of exosomes similar to heat-shocked parasites (Silverman et al. 2010a), two phenotypic characteristics that indicate increased homeostatic stress as the likely cause of intracellular cyp40-1- parasite destruction.

\section{MATERIALS AND METHODS}

\section{Ethics statement}

All animals were handled according to institutional guidelines of the Central Animal Facility of the Institut Pasteur (Paris, France) and experiments were performed in accordance with protocols approved by the Animal Experimentation Ethics Committee of the Institut Pasteur and the veterinary service of the French Ministry of Agriculture (number B-75-1159, 30 May 2011). The animals were housed and handled in accordance with good animal practice as defined by FELASA (www.felasa.eu/guidelines.php).

\section{Mice, macrophages and parasites}

Wild-type C57BL/6 mice were purchased from Charles River Laboratories (Wilmington, MA), and cytochrome B245 (NADPH oxidase) null mutant (Phox-/-) B6.129SCybb $b^{\text {tm/Din }} / \mathrm{J}$ mice (Pollock et al. 1995) and inducible NO synthase null mutant (iNOS-/-) B6.129P2-Nos $2^{\text {tm/Lau }} / \mathrm{J}$ mice (Laubach et al. 1995) were purchased from the Jackson Laboratory (Bar Harbor, ME). Mice were kept and treated according to the institutional guidelines for animal experiments. Bone marrow-derived macrophages (BMMs) were prepared from the femurs of 6-8 wks old female mice as described (Forestier et al. 2011). Leishmania donovani strain 1SR (MHOM/SD/62/1SR) was maintained at $25{ }^{\circ} \mathrm{C}, \mathrm{pH} 7.4$ in supplemented M199 medium (Thermo Fisher Scientific, Waltham, MA) (Hubel et al. 1997; Kapler et al. 1990). Axenic differentiation into amastigotes was performed as described (Goyard et al. 2003; Morales et al. 2008). cyp40-/- null mutant parasites were generated as previously described by sequential replacement of the endogenous CYP4O alleles using two targeting constructs comprising puromycin and bleomycin resistance genes flanked by approximately 1,000 bp of the $5^{\prime}$ and $3^{\prime}$ UTRs of the L. infantum CYP4O gene (GeneDB accession number LinJ.35.4830; Yau et al. 2014). For genetic complement of cyp40-/- null mutants, a cyp40-/-/+ "add-back" line was established using a knock in strategy integrating the CyP40 ORF and 3'UTR into the ribosomal locus (Yau et al. 2014).

\section{Macrophage infection assays}

Isolation and differentiation of BMMs were performed as described (Forestier et al. 2011). Briefly, the dislodged mature BMMs were plated overnight in 24-well plates containing sterile 12-mm glass coverslips at a cell density of $1 \times 10^{5}$ cells/well. Leishmania donovani promastigotes cultured for $2 \mathrm{~d}$ at stationary phase $\left(8-10 \times 10^{7} / \mathrm{ml}\right)$ were washed three times with plain RPMI medium (Thermo Fisher Scientific) and once with infection medium $(0.7 \%$ 
BSA, $25 \mathrm{mM}$ HEPES [pH 7.4] in plain RPMI medium), incubated with BMMs at $37{ }^{\circ} \mathrm{C}$ with $5 \% \mathrm{CO}_{2}$ for $2 \mathrm{~h}$ at a multiplicity of infection of 10 parasites per host cell. Extracellular parasites were then removed by washing the coverslips extensively with PBS (Thermo Fisher Scientific) and transfer of the coverslip into new 24-well plates containing $1 \mathrm{ml}$ of fresh complete RPMI medium per well. This washing procedure was repeated daily until $48 \mathrm{~h}$ post infection. The plates were incubated for up to $48 \mathrm{~h}$ at $37{ }^{\circ} \mathrm{C}$ with $5 \% \quad \mathrm{CO}_{2}$ during the experiment. For mixed infection study, cytoplasmic labeling of parasites with carboxyfluorescein diacetate succinimidyl ester (CFSE; Thermo Fisher Scientific) was performed as described (Morales et al. 2010).

\section{Analysis of intracellular parasite burden}

Counting of macrophages and intracellular parasites was performed as described (Yau et al. 2014). Briefly, 4\% (w/v) paraformaldehyde-fixed macrophage-attached coverslips were mounted and stained with Mowiol medium containing Hoechst 33342 (Thermo Fisher Scientific). At least 100 macrophages were imaged per coverslip using Axioplan 2 wide field light microscope with the Apotome module (Carl Zeiss AG, Oberkochen, Germany). Number of parasites and macrophages was estimated by counting macrophage and parasite nuclei using ImageJ (National Institute of Health, Bethesda, MD).

\section{Transmission electron microscopy}

Parasites were washed twice in ice-cold PBS and then fixed with $2 \%(v / v)$ glutaraldehyde (Sigma-Aldrich, St. Louis, MO) in PBS with $0.1 \mathrm{M}$ sodium cacodylate $(\mathrm{pH}$ 7.2). The fixed cells were postfixed in $1 \%(\mathrm{w} / \mathrm{v}) \mathrm{OsO}_{4}$ and then dehydrated with increasing ethanol concentrations (70-100\%) and embedded in EPON epoxy resin (Hexion Speciality Chemicals, Columbus, $\mathrm{OH}$ ). Ultrathin sections $(\sim 70 \mathrm{~nm})$ were prepared using an Ultramicrotome (Ultra Cut E; Reichert/Leica, Depew, NY) and stained with uranyl acetate and lead citrate. Sections were analyzed using the Tecnai Sprit TEM (FEl, Hillsboro, OR).

\section{Exosome isolation}

Exosomes were purified from L. donovani WT, cyp40-1or cyp40-1-/+ axenic amastigote culture supernatant as described previously (Lambertz et al. 2015; Silverman et al. 2010a). Briefly, $200 \mathrm{ml}$ of day 5 promastigotes (at a concentration of $5 \times 10^{7}$ cells $/ \mathrm{ml}$ ) were transformed into amastigotes by washing $2 X$ with Hank's buffered salt solution (HBSS; Sigma-Aldrich) followed by incubation in serum-free buffered exosome collection media at $\mathrm{pH} 5.5$ (RPMI1640 supplemented with 1\% D-glucose, $20 \mathrm{mM}$ HEPES, $2 \mathrm{mM}$ L-glutamine, $100 \mathrm{U} / \mathrm{ml}$ penicillin/streptomycin and $25 \mathrm{mM} \mathrm{MES}$, all from Sigma-Aldrich), at $37^{\circ} \mathrm{C}$ for $24 \mathrm{~h}$. Exosomes were purified from culture supernatants by a series of centrifugation and filtration steps, followed by flotation on a sucrose cushion, as described (Silverman et al. 2010a).

\section{Nanosight analysis}

The size and concentration of the isolated exosomes were measured using the NanoSight ${ }^{\mathrm{TM}}$ LM10-HS10 system (Malvern Instruments, Worcestershire, UK). For analysis, a monochromatic laser beam $(405 \mathrm{~nm})$ was applied to the diluted exosome solution (1:1,000 in 0.02 $\mu \mathrm{m}$-filtered PBS) that was injected into a LM12 viewing unit using a computer controlled syringe pump. NanoSight ${ }^{\mathrm{TM}}$ tracking analysis (NTA) software version 2.3 was used to produce the mean and median vesicle size together with an estimate of particle concentration. Samples were measured three times to confirm reproducibility.

\section{D-DIGE ANALYSIS}

Total proteins from stationary phase L. donovani WT and cyp40-/- were analyzed with quantitative 2D-DiGE (Pescher et al. 2011). Proteins from stationary phase L. donovani WT and cyp40-/- were precipitated using the 2D Clean-Up kit (Bio-Rad Laboratories, Hercules, CA). Pellets were resuspended in DIGE sample buffer $(7 \mathrm{M}$ Urea, $2 \mathrm{M}$ Thiourea, 4\% CHAPS, $30 \mathrm{mM}$ Tris/ $\mathrm{HCl}$, $\mathrm{pH}$ 8.5) to a final protein concentration of $5 \mathrm{mg} / \mathrm{ml}$, and differentially labeled with the spectrally resolvable G-Dye200 and G-Dye300. A pool of both extracts was labeled with G-Dye100 for normalization purposes (DyeAgnostics, Halle, Germany). $150 \mu \mathrm{g}$ of protein containing $50 \mu \mathrm{g}$ of G-Dye200, G-Dye300, G-Dye100 labeled samples were pooled together and adjusted with $150 \mu$ Destreak rehydration buffer (GE Healthcare, Chicago, IL) containing 0.5\% IPG buffer 4-7 and 1.0\% DTT. Samples were separated in the first dimension overnight at $20{ }^{\circ} \mathrm{C}$ using the gel-based IPGphor isoelectric focusing system (GE Healthcare) and $18 \mathrm{~cm}$ DryStrip pH 4-7 immobiline strips. Strips were rehydrated overnight at RT in Destreak rehydration solution containing $0.5 \%$ IPG Buffer 4-7. Samples were applied to the acidic end of the gel using a sample cup. The maximum current setting was $50 \mu \mathrm{A} /$ strip and IEF run was carried out using the following conditions: $500 \mathrm{~V}$ gradient step for $1 \mathrm{~h}, 1,000 \mathrm{~V}$ gradient step for $1 \mathrm{~h}, 2,000 \mathrm{~V}$ gradient step for $4 \mathrm{~h}, 4,000 \mathrm{~V}$ gradient step for $2 \mathrm{~h}$, $8,000 \mathrm{~V}$ for $11.5 \mathrm{~h}$ and $8,000 \mathrm{~V}$ for $2 \mathrm{~h}(98,250 \mathrm{Vh})$. Following IEF, strips were sequentially incubated for $15 \mathrm{~min}$ in $6 \mathrm{M}$ urea, $75 \mathrm{mM}$ Tris/ $\mathrm{HCl} \mathrm{pH} 8.8,29.3 \%$ glycerol, 4\% SDS, $0.002 \%$ bromophenol blue supplemented with $65 \mathrm{mM}$ DTT, and bromophenol blue supplemented with $13.5 \mathrm{mM}$ iodoacetamide. The strips were transferred to SDS polyacrylamide gels and sealed with $0.5 \%$ agarose in 25 mM Tris-base, $0.19 \mathrm{M}$ glycine, 0.2\% SDS, 0.01\% bromophenol blue. Electrophoresis was carried out in an Ettan DALT six electrophoresis system (GE Healthcare) using $12.5 \%$ SDS-PAGE gels and two-step runs $(1 \mathrm{~W} / \mathrm{gel}$ for $1 \mathrm{~h}$ and $2 \mathrm{~W} / \mathrm{gel}$ for $16 \mathrm{~h}$ ).

2D-DiGE image acquisition and protein identification. Gels were scanned on a Typhoon ${ }^{\mathrm{TM}}$ Variable Mode Imager 9400 (GE Healthcare) at $532 \mathrm{~nm}$ (580 BP 30/green), $633 \mathrm{~nm}$ (670 BP 30/red) and $488 \mathrm{~nm}$ (520 BP 40/blue). Quantitative analysis of the images was performed using 
the DeCyder ${ }^{\mathrm{TM}}$ 2D Differential Analysis Software (GE Healthcare). Spots showing a minimum of 1.5-fold change in fluorescence intensity with a $p$-value $<0.01$ were considered significantly modulated. After staining with Lava purple (Fluorotechnics, Gladesville, Australia), 2D gel spots of interest were excised from gels using the ProPic Investigator robotic system (Genomic Solutions, Ann Arbor, MI) and collected in 96-well plate. Destaining, reduction, alkylation, and trypsin digestion of the proteins followed by peptide extraction were carried out with the ProGest Investigator (Genomic Solutions). MS analysis was carried out on the 4800 Proteomics Analyzer (Applied Biosystems, Waltham, MA) and analyzed by GPS Explorer 2.0 software (Applied Biosystems/MDS SCIEX) using a database of the $\mathrm{NCBI}$ no. 20100119 (10348164 sequences; 3529470745 residues). Protein hits with MASCOT Protein score $\geq 50$ and a GPS Explorer Protein confidence index $\geq 95 \%$ were used for further manual validation.

\section{SDS-PAGE and Western blotting}

Total cell lysate (TCL) of Leishmania was prepared by lysing $1.25 \times 10^{9}$ parasites per $\mathrm{ml}$ of lysis buffer $(20 \mathrm{mM}$ Tris- $\mathrm{HCl} \mathrm{pH} 7.5,150 \mathrm{mM} \mathrm{NaCl}, 1 \%$ Triton $X-100,1 \mathrm{mM}$ EDTA, $2.5 \mathrm{mM}$ sodium orthovanadate, $1 \mathrm{mM} \beta$-glycerophosphate, $1 \mathrm{mM}$ PMSF, $5 \mu \mathrm{g} / \mathrm{ml}$ aprotinin, $5 \mu \mathrm{g} / \mathrm{ml}$ leupeptin). Lysates were incubated on ice for $5 \mathrm{~min}$, followed by addition of $4 \mathrm{X}$ Laemmli loading buffer and boiling for 7 min. Lysates were then cleared by centrifuging at $13,000 \mathrm{~g}$ for $5 \mathrm{~min}$. Exosome lysates were prepared by directly adding $4 \mathrm{X}$ Laemmli loading buffer and $1 \mathrm{mM}$ PMSF to purified exosomes (in $5 \mathrm{mM}$ HEPES and $150 \mathrm{mM} \mathrm{NaCl}$ ), followed by boiling and centrifuging as above. Samples were run on 10\% SDS-PAGE gels, followed by transfer to nitrocellulose membranes or silver staining. Immunoblotting was performed by blocking membranes with 3\% dry milk powder in PBS for 45 min at room temperature, followed by incubation with primary antibodies against EF1- $\alpha$ (clone CBP-KK1; Millipore Merck KGaA, Darmstadt, Germany) diluted 1:1,500 in 3\% milk in PBS for $2 \mathrm{~h}$ at room temperature. After several washes, blots were incubated with secondary antibodies (antimouse-Alexa680 from Life Technologies, Carlsbad, CA) for $1 \mathrm{~h}$ at room temperature, washed again and scanned with the Odyssey CLx infrared imaging system (LI-COR, Lincoln, NE). Alternatively, blots were probed with chicken IgY against HSP100, HSP90 or HSP70 (Hubel et al. 1995), followed by reaction with anti-chicken IgY-alkaline phosphatase and development using NBT and X-phosphate. Band intensities were measured using the ImageJ software (NIH) and normalized against Coomassie Bluestained SDS-PAGE gels.

\section{Silver staining}

Silver staining of proteins separated by SDS-PAGE was performed by fixing gels for $1 \mathrm{~h}$ in $50 \% \mathrm{MeOH}$ and $20 \%$ acetic acid in $\mathrm{ddH}_{2} \mathrm{O}$ (fixing solution), followed by three washes with $35 \% \mathrm{EtOH}$ for $20 \mathrm{~min}$. Gels were then sensitized in $0.02 \%$ sodium thiosulfate for $2 \mathrm{~min}$, washed three times with $\mathrm{ddH}_{2} \mathrm{O}$ for 5 min each, followed by incubation in $0.2 \% \mathrm{AgNO}_{3}$ and $0.08 \%$ formaldehyde in $\mathrm{ddH}_{2} \mathrm{O}$ in the dark for 20 min. After two rinses with $\mathrm{ddH}_{2} \mathrm{O}$, gels were developed in $6 \% \mathrm{Na}_{2} \mathrm{CO}_{3}, 0.05 \%$ formaldehyde and $0.0004 \%$ sodium thiosulfate in $\mathrm{ddH}_{2} \mathrm{O}$ until the staining had reached the desired intensity. The reaction was stopped with fixing solution and gels were washed two times in $\mathrm{ddH}_{2} \mathrm{O}$ for 10 min and imaged.

\section{Cell viability}

Parasite viability was determined by Alamar blue assay (Mikus and Steverding 2000). Twenty microliters of $0.01 \%$ $(\mathrm{w} / \mathrm{v})$ resazurin solution was added to the $100 \mu \mathrm{l}$ of cells in a 96-well plate and incubated for $4 \mathrm{~h}$ at $37^{\circ} \mathrm{C}$. Fluorescence intensity was measured $\left(\lambda_{\mathrm{ex}}=560 \mathrm{~nm}\right.$; $\lambda_{\text {em }}=590 \mathrm{~nm}$ ) using microplate reader Xenius XML (Safas, Mo, Monaco). Viability was expressed in \% of fluorescence intensity compared to untreated control. The tests were performed in quadruplicate.

\section{Statistical analysis}

Numerical data were expressed as mean \pm standard deviation. Mann-Whitney $U$ test was used to compare the significance between specific groups and $p<0.05$ was considered as statistically significant. All statistical analysis was performed using either Excel 2003 (Microsoft) or Prism 5.0 (GraphPad Software, La Jolla, CA).

\section{RESULTS}

\section{Cyp40-I- virulence attenuation is independent of host cell reactive oxidant and nitrogen species}

We previously revealed an important role for the $L$. donovani putative cochaperone cyclophilin 40 during the parasite's intracellular life cycle stage by loss-of-function analysis (Yau et al. 2014). The functional role(s) of CyP40 in virulence remained elusive, however. Here, we investigate if the abrogated intracellular survival of null mutant parasites is the result of an increased susceptibility to macrophage antimicrobial activities. We first tested parasites in vitro for resistance against challenges that mimic the cytotoxic environment encountered inside host cells. Stationary parasites of WT, cyp40-/- and cyp40-/-/+ were incubated with increasing amounts of $\mathrm{H}_{2} \mathrm{O}_{2}$ or trypsin. Parasite viability compared to untreated controls was determined by the Alamar blue assay. Both WT and cyp40-1- parasites did not show any significant difference in response to the challenge with either agent, suggesting that CyP40 is not required for parasite resistance to oxidative stress or proteolytic attack in culture (Fig. 1A).

To further investigate the role of reactive oxidant species (ROS) and leishmanicidal nitric oxide (NO) in the elimination of cyp40-/- parasites, we chose a physiologically more relevant setting, tracking parasite survival within macrophages obtained from Phox-/- or iNOS-/- mutant 


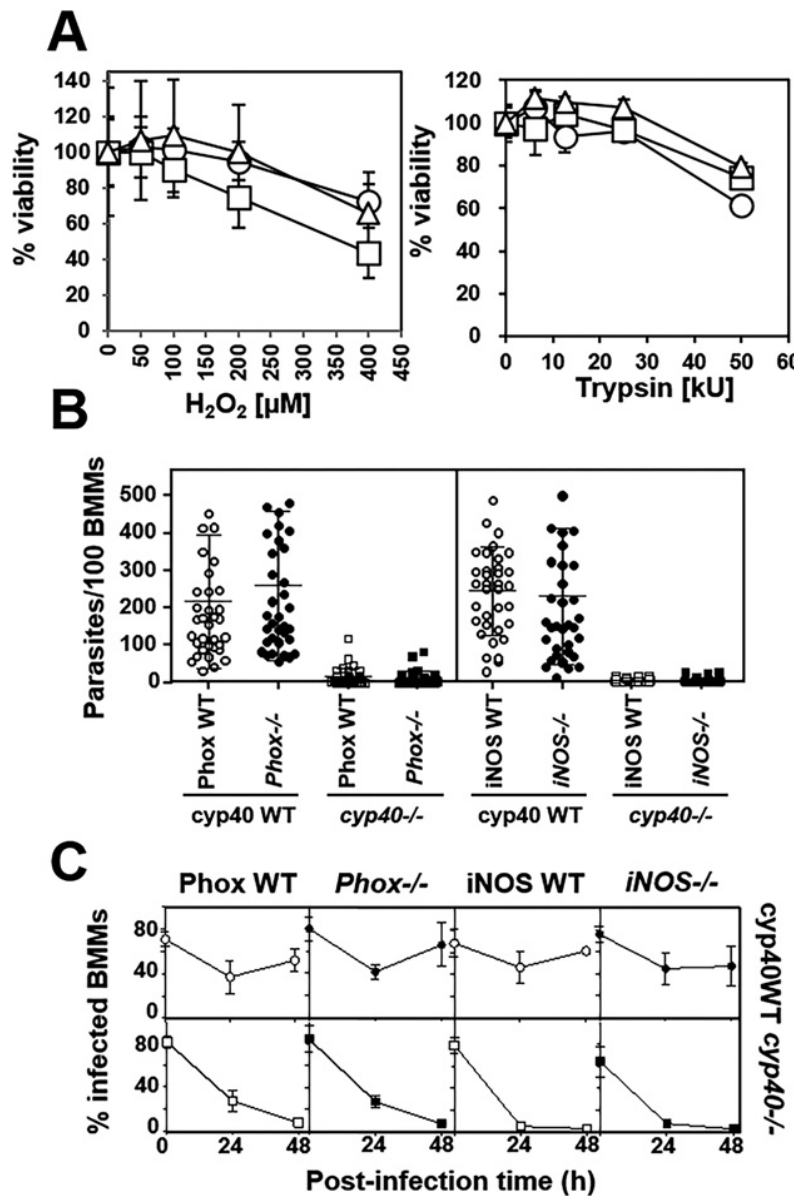

Figure 1 Macrophage infection assays with CYP40 null mutant parasites. (A) In vitro susceptibility of CYP40 null mutant parasites to oxidants and protease. Logarithmic promastigotes of wild-type (WT) (circles), cyp40-1- (open squares), and cyp40-/-/+ addback (triangle) were incubated with either hydrogen peroxide $\left(\mathrm{H}_{2} \mathrm{O}_{2}\right.$, upper panel) or trypsin (lower panel) at $25^{\circ} \mathrm{C}, \mathrm{pH} 7.4$ for $48 \mathrm{~h}$. Cell viability was assessed by Alamar blue assay as described in experimental procedures. Experiments were performed in quadruplicate and \% viability \pm standard deviation is presented. (B, C) CYP40 null mutant parasites infection to oxidant-deficient macrophages. Bone marrow-derived macrophages (BMMs) from C57BL/6 WT (B6), Phox-/- (P-/-) and iNOS $-/-(\mathrm{N}-/-)$ mice were infected with stationary promastigotes of Leishmania donovani WT (CyP40WT) or cyp40-/- (CyP40KO). Percent infected BMMs during $48 \mathrm{~h}$ post-infection period (panel B) and parasites/100 BMMs at $48 \mathrm{~h}$ post-infection (panel C) are shown. O, C57BL/6 WT macrophages infected with CyP40WT; •, C57BL/6 Phox-/- or iNOS-1- mutant macrophages infected with CyP40WT. 口, C57BL/6 WT macrophages infected with CyP40KO;, C57BL/6 Phox-1- or iNOS-1- mutant macrophages infected with CyP4OKO. Error bars represent standard deviation of one triplicate experiment.

mice that lack ROS or NO activities respectively. Bone marrow precursor cells were differentiated into macrophages in vitro, incubated with WT and cyp40-/- stationary phase promastigotes for $4 \mathrm{~h}$, and after removal of free parasites, intracellular parasite burden was monitored by fluorescence microscopy at 0, 24, and $48 \mathrm{~h}$ post-infection. WT parasites persisted at high levels during the 48-h infections in WT as well as Phox-/- and iNOS-/- macrophages (Fig. 1B, C). As expected, cyp40-/- parasites failed to establish intracellular infection in WT macrophages and were largely eliminated at $48 \mathrm{~h}$ postinfection. cyp40-1- survival was not restored in either Phox-/- or iNOS-1- macrophages. Thus, the cyp40-/- defect in intracellular survival was not compensated in either of the mutant host cells, ruling out the corresponding activities as the major causes for intracellular parasite death.

\section{Co-infection with wild-type parasites does not restore cyp40-/- infectivity}

We next tested if cyp40-1- survival can be rescued in trans by the immune-subversive activity of virulent WT parasites. WT and cyp40-1- promastigotes from stationary growth phase cultures were differentially labeled with the cytoplasmic dye carboxyfluorescein diacetate succinimidyl ester (CFSE) (Morales et al. 2010). Unlabeled WTand CFSE-labeled cyp40-/- promastigotes were mixed at a ratio of $1: 1$, and intracellular parasite survival was monitored by fluorescence microscopy in infected BMMs using nuclear and cytoplasmic staining as read outs. As determined by fluorescence microscopy, our assay efficiently distinguished between WT and cyp40-/- parasites during mixed in vitro infection (Fig. 2, upper panel). Over the $48 \mathrm{~h}$ post-infection period, neither WT survival nor cyp40-/- elimination was significantly affected in superinfected macrophages (Fig. 2, lower panel). These data suggest that the intracellular killing of null mutant parasites is not dependent on the main host cell cytotoxic activities that are known to be suppressed by infectious WT parasites. Conversely, as deduced from WT parasite survival during mixed infection, cyp40-/- parasites did not trigger increased leishmanicidal activities in macrophages. Hence, intracellular destruction of cyp40-1occurs independently from the host cytotoxic activities that are suppressed or subverted by virulent parasites. Together these results point toward a cis-acting, parasiteintrinsic defect in intracellular viability as the cause of parasite virulence loss, a possibility that we further explored in the following.

\section{The lack of CyP40 expression is compensated by increased HSP expression}

We gained a first insight into potential homeostatic changes caused by the loss of CyP40 expression using proteomic profiling. Protein abundance was compared by quantitative two dimensional differential gel electrophoresis (2D-DiGE) using total protein extracts obtained from stationary phase cyp40-/- and WT parasites (Fig. 3A). Aside from the previously published up-regulation of a tubulin isoform (LinJ.21.2240; Yau et al. 2014), cyp40-/parasites showed a statistically significant 1.5-2.3-fold upregulation of various heat shock proteins and chaperones, including the cytoplasmic HSP70 family member LinJ.26.1220 (spot 386), the putative chaperonin containing t-complex proteins LinJ.32.1060 (spot 425) and 


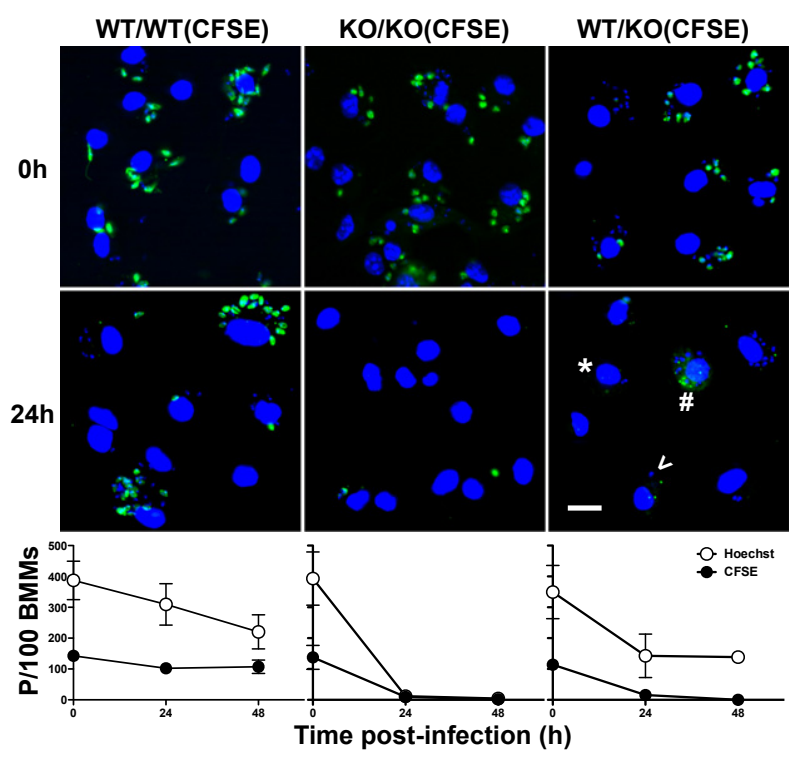

Figure 2 Macrophage infection assays with mixed parasite population. Leishmania donovani wild-type (WT) or cyp40-/- promastigotes were prelabeled with CFSE. Bone marrow-derived macrophages (BMMs) from C57BL/6 mice were then infected in the following combinations: (i) nonlabeled WT plus CFSE-labeled WT, (left panel) (ii) nonlabeled cyp40-/-plus CFSE-labeled cyp40-/- (middle panel), and (iii) nonlabeled WT plus CFSE-labeled cyp40-1- (right panel). The two different parasite populations in each combination were mixed at a ratio of $1: 1$ and incubated with $\mathrm{BMMs}$ at $37{ }^{\circ} \mathrm{C}$ with $5 \% \mathrm{CO}_{2}$ for $2 \mathrm{~h}$. At 0 , 24 , and $48 \mathrm{~h}$ post-infection, BMMs were collected and nuclear staining was performed for parasite burden analysis. (Upper panel) Fluorescence microscopy of mixed infections at 0 and $24 \mathrm{~h}$ post-infection. *, nuclei of BMMs; \#, CFSE-labeled parasites; <, nuclei of parasites. Scale bars represent $10 \mu \mathrm{m}$. (Lower panel) Burdens of CFSE-labeled parasites of at least $100 \mathrm{BMMs}$ at 0,24 , and $48 \mathrm{~h}$ postinfection are shown with error bars representing standard deviation of one representative triplicate experiment. Note that the Hoechst staining does not discriminate between WT and cyp40-1-.

LinJ.36.7240 involved in protein folding in higher eukaryotes (spot 505), and the hypothetical protein LinJ.14.0460 (spot 848) with similarity to the small heat shock protein HSP20 (Fig. 3B). Western blot analyses of wild type and null mutant promastigotes from logarithmic and stationary growth phases showed no significant difference in abundance of the major heat shock proteins HSP100, HSP90, or HSP70 (Fig. 3C). Conceivably, up-regulation of the identified stress proteins in cyp40-/- may be a compensatory response to the lack of CyP40 activities, either directly substituting for CyP40 stress functions or counteracting cellular stress caused by the absence of CyP40.

\section{Cyp40-I- parasites show vesicle accumulation inside the flagellar pocket}

To further investigate potential cyp40-/- defects we performed ultra-structural analysis of WT, cyp40-/- and cyp40-/-/+ parasites from stationary growth phase by transmission electron microscopy (TEM). Despite the

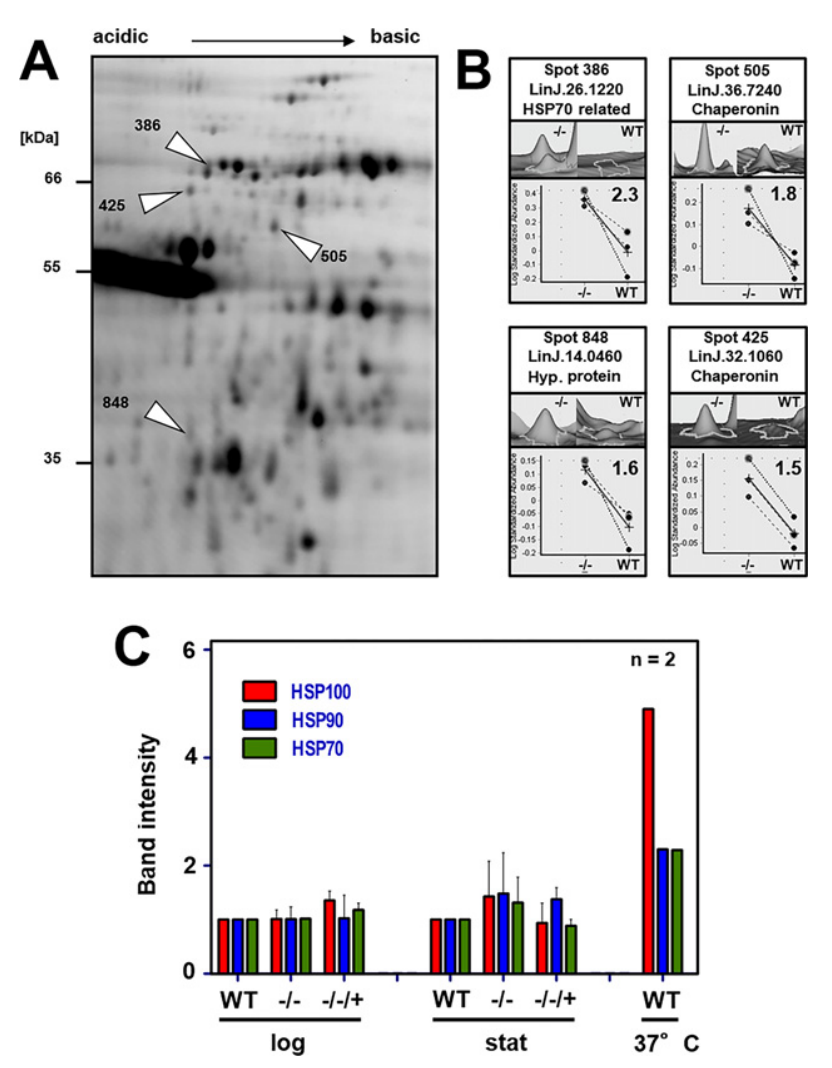

Figure 3 Proteomics analysis of the CyP40 null mutant. (A) 2D-DIGE analysis. $50 \mu \mathrm{g}$ of G-Dye-labeled total protein extracts from wild-type (WT) and cyp40-/- promastigotes from stationary growth phase were separated in the first dimension by IEF on $18 \mathrm{~cm} \mathrm{pH} \mathrm{4-7} \mathrm{IPG} \mathrm{strips,} \mathrm{and}$ the second dimension by SDS-PAGE. Four gels representing independent experimental replicates were scanned with a Typhoon imager and analyzed with the DeCyder ${ }^{\mathrm{TM}}$ software package from GE Healthcare. Gels were then stained with Lava purple, revealed by scanning on a Typhoon imager, and differentially expressed proteins were subjected to MS analysis. Spots of interest are labeled with ID numbers. Molecular weight markers in kDa are indicated. (B) Biological Variation Analysis. The readout of the DeCyder Biological Variation Analysis (BVA) module is shown for four proteins that show differential abundance. Spot number, accession number, and annotation are given as on the GeneDB web site (www.genedb.org) (upper panels). The middle panels represent 3D images of the volumes of spots of interest from WT and cyp40-1- (-/-). The bottom panels show graphic representations of differences in abundance of these proteins across three independent experiments. The numbers indicate the fold difference in abundance of cyp40-1- to WT. (C) Western blot analysis. Promastigotes of WT, cyp40-1- and cyp40-/-/+ genotype were collected either from synchronized, logarithmically growing cultures (log), or from cultures after $2 \mathrm{~d}$ in stationary growth phase (stat). Wild-type promastigotes exposed to $37{ }^{\circ} \mathrm{C}$ were tested as controls. $10 \mu$ of total cell extract (corresponding to $1.3 \times 10^{7}$ ), or $30 \mu \mathrm{l}$ of exosomal extract (harvested from the supernatant of $9.3 \times 10^{9}$ cells) from each sample were analyzed by SDS-PAGE and Western blot using anti-HSP100, anti-HSP90 and antiHSP70 antibodies. The blots were developed using NBT and X-phosphate. The staining intensities were quantified by ImageJ analysis and normalized first against total protein and then against the wild type results for log or stat parasites. The experiments were done in duplicate and the mean intensities are shown. 
important morphological defects previously observed in cyp40-1- mutants, these parasites did not have any overt effects on sub-cellular and organellar organization, which appeared normal when compared to WT parasites (Fig. 4). However, we noted a striking accumulation of vesicles inside the flagellar pocket of cyp40-1- cyp40-/- parasites that escaped our attention in previous analyses and was not observed in the cyp40-/-/+ addback control, thus revealing a CyP40-specific phenomenon. This observation may either reflect a defect in vesicle release leading to accumulation inside the flagellar pocket, or may result from an increase in the steady-state production and release of vesicles. To distinguish between these two possibilities and to assess if these vesicles correspond to previously described exosomes (Silverman et al. 2010a) we next analyzed the vesicular content of culture supernatants of WT, cyp40-/- and cyp40-/-/+ parasites.

Enhanced exosomal biogenesis in cyp40-/- cells

Exosomes were purified from supernatants of cultures grown to equal cell density using a protocol that was designed to specifically isolate exosomes based on their unique size and density (Lamparski et al. 2002; Silverman et al. 2010a). We performed these experiments using axenic amastigotes rather than stationary promastigotes to recover the necessary amounts of exosomes required for our quantitative and qualitative comparative analyses. Analysis of the obtained fractions by optical light scattering using a NanoSight instrument revealed a significant increase in the number of nanovesicles released by cyp40-1- parasites that corresponded in size to bona fide exosomes (Fig. 5A, B), thus confirming increased vesicle production in cyp40-/- parasites, which seems independent from the in vitro culture stage. Western blot analysis of exosomal fractions for the presence of the exosomal marker protein elongation factor- $1 \alpha(E F-1 \alpha)$ confirmed this finding and repeatedly demonstrated an increased abundance of EF-1 $\alpha$ in cyp40-1- exosomes, suggesting either increased exosome release or enhanced packaging of proteins within exosomes in the mutant parasites (Fig. 5C).

\section{Cyp40-I- exosomes are altered in protein cargo}

Visualization of gel-separated exosomal extracts by silverstaining revealed significant differences in the band
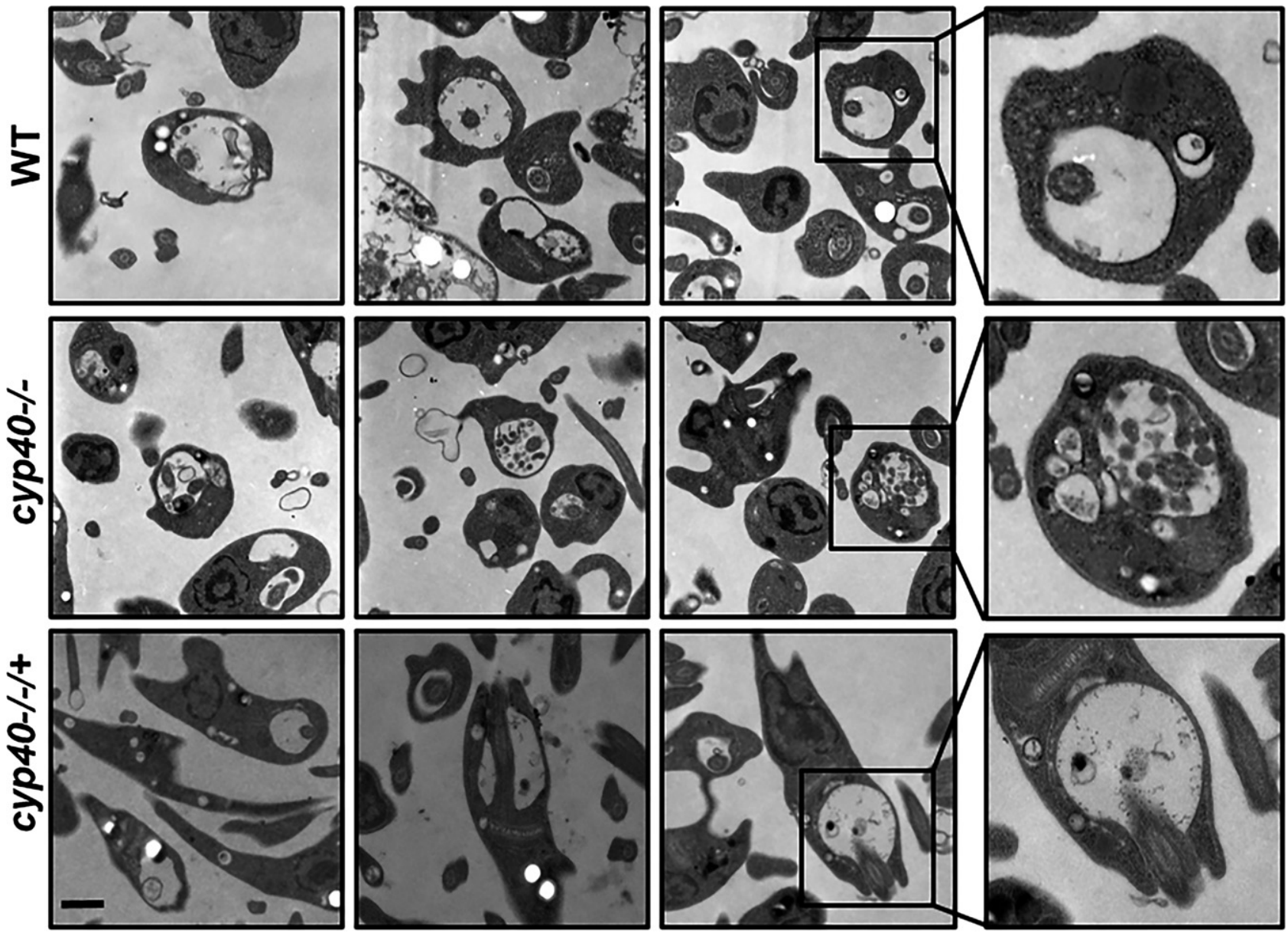

Figure 4 Transmission EM analysis. Promastigotes from stationary growth phase were fixed and processed for transmission EM as described in Materials and Methods. Scale bar represents $500 \mathrm{~nm}$. 
A

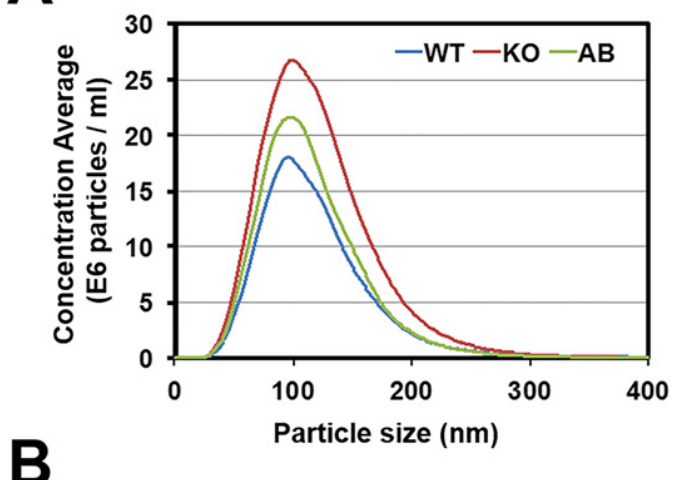

\begin{tabular}{|l|c|c|c|}
\hline Sample & $\begin{array}{c}\text { Size } \\
\text { (mean, } \mathbf{n m})\end{array}$ & $\begin{array}{c}\text { Size } \\
\text { (mode, } \mathrm{nm})\end{array}$ & $\begin{array}{c}\text { Concentration } \\
\left(\mathbf{1 0 ^ { 8 }} \text { particles/ml) }\right.\end{array}$ \\
\hline WT & $119 \pm 0.5$ & $95 \pm 1.5$ & $16.50 \pm 0.21$ \\
\hline cyp40-/- & $121 \pm 0.6$ & $98 \pm 0.3$ & $26.65 \pm 0.88$ \\
\hline cyp40-/-/+ & $116 \pm 0.8$ & $95 \pm 2.3$ & $19.59 \pm 0.74$ \\
\hline
\end{tabular}

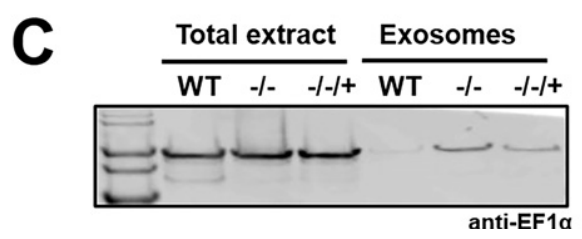

Figure 5 Quantitative exosome analysis. Leishmania donovani wildtype (WT), cyp40-1- (-/-, or knock out KO) and cyp40-/-/+ (-/-/+, or addback $\mathrm{AB}$ ) were transformed into axenic amastigotes. Exosomes released into culture supernatants were collected and purified. Size and particle concentration of purified exosomes (diluted 1:1,000 with PBS) were analyzed by nanoparticle tracking analysis with Nanosight (A, the corresponding quantitative data are shown in B). (C) Western blot analysis. Equal volumes of total cell extract and exosome lysates were separated by SDS-PAGE, followed by transfer to nitrocellulose and Western Blotting with anti-EF1 $\alpha$. The images are representative of two independent experiments.

pattern in cyp40-/- compared to WT and addback control, suggesting that both amount and cargo of exosomes are altered in cyp40-/- parasites (Fig. 6A). In particular we identified one prominent signal specific for the null mutant at approximately $20 \mathrm{kDa}$. Proteomics analysis identified numerous comigrating proteins, including the cochaperone GrpE that was discovered with the highest coverage rate of over 38\% (Fig. 6B). Whether this protein represents the bulk of the signal and accumulates in cyp40-1- exosomes in compensation for the absence of CyP40 remains to be elucidated.

\section{DISCUSSION}

Using a null mutant approach we previously demonstrated that the Leishmania immunophilin protein family member CyP40 does not affect parasite viability in culture but is

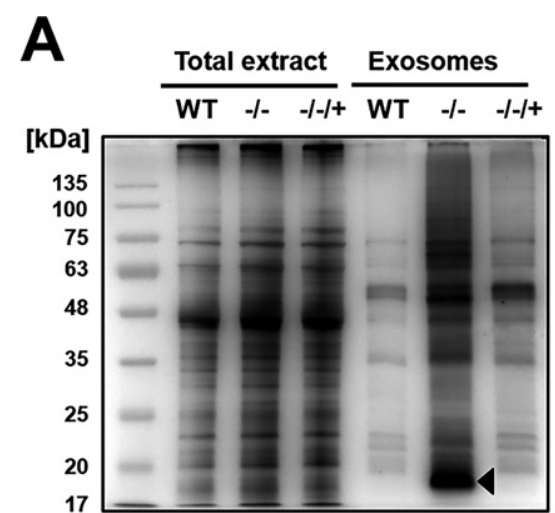

B

\begin{tabular}{|l|c|c|l|}
\hline Gene ID & $\%$ & MW (Da) & annotation \\
\hline LDBPK_300770 & 38.5 & 22645 & co-chaperone, GrpE, putative \\
\hline LDBPK_242280 & 37.2 & 26603 & Hypothetical protein, conserved \\
\hline LINJ_16_1200 & 28.9 & 29069 & Hypothetical protein, conserved \\
\hline LINJ08_0310 & 28.2 & 28296 & $\begin{array}{l}\text { Hypothetical protein, conserved } \\
\text { Isochorismatase family, putative }\end{array}$ \\
\hline LINJ_36_6990 & 22.2 & 18607 & Hypothetical protein, conserved \\
\hline
\end{tabular}

Figure 6 Qualitative exosome analysis. Leishmania donovani wildtype (WT), cyp40-1- $(-/-)$ and cyp40-1-/+ $(-1-1+)$ were transformed into axenic amastigotes. Exosomes released into culture supernatants were collected and purified. (A) $3 \mu$ of total cell lysate (corresponding to $4 \times 10^{6}$ cells), or $10 \mu \mathrm{l}$ of exosomal extract (harvested from supernatant of $3.1 \times 10^{9}$ cells were separated by SDSPAGE, followed by silver staining and identification of proteins in the indicated signal (arrowhead) by mass spectrometry. (B) Results of the MS analysis. \%, percent peptide coverage.

required to establish successful macrophage infection (Yau et al. 2014). Here, we analyzed the cyp40-/- mutant phenotype in more detail combining macrophage infection assays, proteomics and ultra-structural analyses, and reveal homeostatic stress as the likely cause for its virulence attenuation thus defining CyP40 as an essential chaperone for intracellular viability.

The capacity of Leishmania to cause disease is linked to a complex interplay between the parasite stress response, cell cycle regulation, and its differentiation into various life cycle stages that are adapted for survival and proliferation in the sand fly insect vector and mammalian host (Wiesgigl and Clos 2001). Inside the sand fly, noninfectious procyclic promastigotes differentiate into highly virulent, cell cycle arrested metacyclic parasites (Sacks and Perkins 1984) in response to changes in $\mathrm{pH}$ and nutritional stress (Cunningham et al. 2001; Franke et al. 1985; Zakai et al. 1998). Following transmission and phagocytic uptake into host macrophages, these parasites re-enter the cell cycle and concomitantly differentiate into amastigotes in response to acidic $\mathrm{pH}$ and temperature shock encountered inside the fully acidified phagolysosome of the host cell (Barak et al. 2005; Zilberstein and Shapira 1994). Leishmania survival in both vector and host depends on the parasites' capacity to evade or resist a highly toxic, hydrolaserich and digestive environment through the expression of stage-specific virulence factors (Gregory and Olivier 2005; McCall et al. 2013). As judged by various in vitro assays, 
cyp40-1- parasites show no major defects during stage differentiation or in resistance to various forms of stress. First, cyp40-1- mutants convert into metacyclic-like parasites at stationary growth phase and differentiate normally into axenic amastigotes as judged by the expression of the stage-specific marker proteins SHERP and A2 respectively (Yau et al. 2014). Second, these parasites show WT resistance to a variety of environmental insults in vitro, including acidic $\mathrm{pH}$, temperature shock, oxidative and hydrolytic attack, or nutritional starvation at stationary growth phase.

The lack of an overt stress phenotype in these mutants calls into question the cochaperone function of Leishmania CyP40 that can be deduced from its conserved peptidylprolyl isomerase and TPR domains implicated in protein folding and interaction with HSP70 respectively (Blatch and Lassle 1999; Shaw 2007). However, analysis of the LdCyP40-dependent proteome proves the opposite and clearly defines this molecule as an important stress protein whose absence is compensated by the upregulation of various unrelated chaperones. Indeed, a closer investigation uncovered other signs of homeostatic stress in cyp40-/- parasites. At stationary phase, these mutants show a strong reduction of cell motility and adopt an oval cell shape, both signs previously associated with cellular stress (Barak et al. 2005; Wiesgigl and Clos 2001; Yau et al. 2014). Furthermore, we observed a significant increase in exosome biogenesis in cyp40-/- cells. These nano-sized vesicles are released by various prokaryotic and eukaryotic cells and carry important functions in intercellular communication and host/pathogen interaction (Silverman and Reiner 2011). In Leishmania, the release of exosomes in culture and into infected host cells has been demonstrated, and this process is enhanced in response to temperature shock (Silverman et al. 2010a). In addition, cyp40-/- exosomes showed important alterations in their protein cargo, with accumulation of proteins in the $20 \mathrm{kDa}$ range, including the cochaperone GrpE that acts as nucleotide exchange factor for bacterial DnaK and mitochondrial HSP70 (Liberek et al. 1991; Nakai et al. 1994). Significantly, this protein has not been identified in L. donovani wildtype exosomes (Silverman et al. 2010a). It remains to be elucidated if its vesicular release is a sign of mitophagy, an autophagy-related process observed in yeast cells under stress conditions that limits mitochondrial damage by increased organelle turnover (Muller et al. 2015). Thus, both the quantitative and qualitative changes we observed in exosomal biogenesis in cyp40-1- cells further sustain our hypothesis that these mutants experience important homeostatic stress.

The cyp40-1- phenotype is reminiscent of the phenotype of L. donovani HSP100 null mutants that grow normally in vitro, fail to establish intracellular infection, and show altered exosomal protein contents (Hubel et al. 1997; Krobitsch et al. 1998; Silverman et al. 2010b). In contrast with wildtype exosomes that stimulate an antiinflammatory response in infected macrophages and exosome-treated dendritic cells, hsp100-/- exosomes cause a pro-inflammatory response detrimental to intracellular parasite survival. It is interesting to speculate that CyP40 plays a similar role in the biogenesis of anti-inflammatory exosomes and that changes in the exosomal cargo in cyp40-/- parasites may cause macrophage activation, thus abrogating intracellular infection. However, we can rule out this possibility based on our findings that cyp40-/- parasites were neither rescued in iNOS deficient macrophages nor in host cells co-infected with wildtype parasites.

In conclusion, even though ablation of CyP40 expression in L. donovani was associated with qualitative and quantitative changes in exosomes, our findings do not support a direct role of this protein in the regulation of exosomal biogenesis, but are more compatible with a role of CyP40 in the parasite stress response. Our study reveals important functions of $L$. donovani CyP40 in the maintenance of normal cellular homeostasis under conditions of environmental stress during stationary phase culture and intracellular macrophage infection. It is conceivable that the simultaneous exposures to intrinsic homeostatic stress, extracellular stress imposed by the host environment, and chemical stress encountered inside the acidified phagolysosome are the cause of cyp40-1- cell death during infection.

\section{ACKNOWLEDGMENTS}

We would like to thank Pascal Lenormand from the Institut Pasteur Proteomics Platform PF3 for protein indetification, Dr. Geetanjali Kharmate and Dr. Emma Guns from the Vancouver Prostate Centre UBC for their assistance with the Nanosight analysis, and Sophie Veillault for help in preparing this manuscript. This work was supported by the 7th Framework Programme of the European Commission through a grant to the LEISHDRUG Project (223414) and the French Government's Investissements d'Avenir programme: Laboratoire d'Excellence 'Integrative Biology of Emerging Infectious Diseases' (Grant No. ANR-10$L A B X-62-I B E I D)$ to G.F.S., and fellowships from the Fondation pour la Recherche Médicale (FDT20100920296) and the Leibniz-DAAD programme to W.-L.Y. U.L. and L.C. were supported from the Canadian Institutes of Health Research (MOP\# 125879 to N.E.R.).

\section{LITERATURE CITED}

Barak, E., Amin-Spector, S., Gerliak, E., Goyard, S., Holland, N. \& Zilberstein, D. 2005. Differentiation of Leishmania donovani in host-free system: analysis of signal perception and response. Mol. Biochem. Parasitol., 141:99-108.

Blatch, G. L. \& Lassle, M. 1999. The tetratricopeptide repeat: a structural motif mediating protein-protein interactions. BioEssays, 21:932-939.

Chakraborty, A., Das, I., Datta, R., Sen, B., Bhattacharyya, D., Mandal, C. \& Datta, A. K. 2002. A single-domain cyclophilin from Leishmania donovani reactivates soluble aggregates of adenosine kinase by isomerase-independent chaperone function. J. Biol. Chem., 277:47451-47460.

Cunningham, M. L., Titus, R. G., Turco, S. J. \& Beverley, S. M. 2001. Regulation of differentiation to the infective stage of the protozoan parasite Leishmania major by tetrahydrobiopterin. Science, 292:285-287. 
Forestier, C. L., Machu, C., Loussert, C., Pescher, P. \& Spath, G. F. 2011. Imaging host cell-Leishmania interaction dynamics implicates parasite motility, lysosome recruitment, and host cell wounding in the infection process. Cell Host Microbe, 9:319-330.

Franke, E. D., McGreevy, P. B., Katz, S. P. \& Sacks, D. L. 1985. Growth cycle-dependent generation of complement-resistant Leishmania promastigotes. J. Immunol., 134:2713-2718.

Goyard, S., Segawa, H., Gordon, J., Showalter, M., Duncan, R., Turco, S. J. \& Beverley, S. M. 2003. An in vitro system for developmental and genetic studies of Leishmania donovani phosphoglycans. Mol. Biochem. Parasitol., 130:31-42.

Gregory, D. J. \& Olivier, M. 2005. Subversion of host cell signalling by the protozoan parasite Leishmania. Parasitology, 130 (Suppl.):S27-S35.

Hoffmann, K. \& Handschumacher, R. E. 1995. Cyclophilin-40: evidence for a dimeric complex with hsp90. Biochem. J., 307(Pt 1):5-8.

Hubel, A., Brandau, S., Dresel, A. \& Clos, J. 1995. A member of the $\mathrm{ClpB}$ family of stress proteins is expressed during heat shock in Leishmania spp. Mol. Biochem. Parasitol., 70:107-118.

Hubel, A., Krobitsch, S., Horauf, A. \& Clos, J. 1997. Leishmania major Hsp100 is required chiefly in the mammalian stage of the parasite. Mol. Cell. Biol., 17:5987-5995.

Kapler, G. M., Coburn, C. M. \& Beverley, S. M. 1990. Stable transfection of the human parasite Leishmania major delineates a 30-kilobase region sufficient for extrachromosomal replication and expression. Mol. Cell. Biol., 10:1084-1094.

Krobitsch, S., Brandau, S., Hoyer, C., Schmetz, C., Hubel, A. \& Clos, J. 1998. Leishmania donovani heat shock protein 100. Characterization and function in amastigote stage differentiation. J. Biol. Chem., 273:6488-6494.

Kulkarni, M. M., Karafova, A., Kamysz, W., Schenkman, S., Pelle, R. \& McGwire, B. S. 2013. Secreted trypanosome cyclophilin inactivates lytic insect defense peptides and induces parasite calcineurin activation and infectivity. J. Biol. Chem., 288:8772-8784.

Lambertz, U., Oviedo Ovando, M. E., Vasconcelos, E. J., Unrau, P. J., Myler, P. J. \& Reiner, N. E. 2015. Small RNAs derived from tRNAs and rRNAs are highly enriched in exosomes from both old and new world Leishmania providing evidence for conserved exosomal RNA Packaging. BMC Genom., 16:151.

Lamparski, H. G., Metha-Damani, A., Yao, J. Y., Patel, S., Hsu, D. H., Ruegg, C. \& Le Pecq, J. B. 2002. Production and characterization of clinical grade exosomes derived from dendritic cells. J. Immunol. Methods, 270:211-226.

Laubach, V. E., Shesely, E. G., Smithies, O. \& Sherman, P. A. 1995. Mice lacking inducible nitric oxide synthase are not resistant to lipopolysaccharide-induced death. Proc. Natl Acad. Sci. USA, 92:10688-10692.

Liberek, K., Marszalek, J., Ang, D., Georgopoulos, C. \& Zylicz, M. 1991. Escherichia coli DnaJ and GrpE heat shock proteins jointly stimulate ATPase activity of DnaK. Proc. Natl Acad. Sci. USA, 88:2874-2878.

Matrangolo, F. S., Liarte, D. B., Andrade, L. C., de Melo, M. F., Andrade, J. M., Ferreira, R. F., Santiago, A. S., Pirovani, C. P., Silva-Pereira, R. A. \& Murta, S. M. 2013. Comparative proteomic analysis of antimony-resistant and -susceptible Leishmania braziliensis and Leishmania infantum chagasi lines. Mol. Biochem. Parasitol., 190:63-75.

McCall, L. I. \& McKerrow, J. H. 2014. Determinants of disease phenotype in trypanosomatid parasites. Trends Parasitol., 30:342-349.

McCall, L. I., Zhang, W. W. \& Matlashewski, G. 2013. Determinants for the development of visceral leishmaniasis disease. PLoS Pathog., 9:e1003053.
Mikus, J. \& Steverding, D. 2000. A simple colorimetric method to screen drug cytotoxicity against Leishmania using the dye Alamar Blue. Parasitol. Int., 48:265-269.

Morales, M. A., Pescher, P. \& Spath, G. F. 2010. Leishmania major MPK7 protein kinase activity inhibits intracellular growth of the pathogenic amastigote stage. Eukaryot. Cell, 9:22-30.

Morales, M. A., Watanabe, R., Laurent, C., Lenormand, P., Rousselle, J. C., Namane, A. \& Spath, G. F. 2008. Phosphoproteomic analysis of Leishmania donovani pro- and amastigote stages. Proteomics, 8:350-363.

Moro, A., Ruiz-Cabello, F., Fernandez-Cano, A., Stock, R. P. \& Gonzalez, A. 1995. Secretion by Trypanosoma cruzi of a peptidyl-prolyl cis-trans isomerase involved in cell infection. EMBO J., 14:2483-2490.

Mukherjee, D., Patra, H., Laskar, A., Dasgupta, A., Maiti, N. C. \& Datta, A. K. 2013. Cyclophilin-mediated reactivation pathway of inactive adenosine kinase aggregates. Arch. Biochem. Biophys., 537:82-90.

Muller, M., Lu, K. \& Reichert, A. S. 2015. Mitophagy and mitochondrial dynamics in Saccharomyces cerevisiae. Biochim. Biophys. Acta, 1853:2766-2774.

Nakai, M., Kato, Y., Ikeda, E., Toh-e, A. \& Endo, T. 1994. Yge1p, a eukaryotic Grp-E homolog, is localized in the mitochondrial matrix and interacts with mitochondrial Hsp70. Biochem. Biophys. Res. Commun., 200:435-442.

Pescher, P., Blisnick, T., Bastin, P. \& Spath, G. F. 2011. Quantitative proteome profiling informs on phenotypic traits that adapt Leishmania donovani for axenic and intracellular proliferation. Cell. Microbiol., 13:978-991.

Pollock, J. D., Williams, D. A., Gifford, M. A., Li, L. L., Du, X., Fisherman, J., Orkin, S. H., Doerschuk, C. M. \& Dinauer, M. C. 1995. Mouse model of X-linked chronic granulomatous disease, an inherited defect in phagocyte superoxide production. Nat. Genet., 9:202-209.

Pratt, W. B., Galigniana, M. D., Harrell, J. M. \& DeFranco, D. B. 2004. Role of hsp90 and the hsp90-binding immunophilins in signalling protein movement. Cell. Signal., 16:857-872.

Sacks, D. L. \& Perkins, P. V. 1984. Identification of an infective stage of Leishmania promastigotes. Science, 223:1417-1419.

Shaw, P. E. 2007. Peptidyl-prolyl cis/trans isomerases and transcription: is there a twist in the tail? EMBO Rep., 8:40-45.

Silverman, J. M., Clos, J., de'Oliveira, C. C., Shirvani, O., Fang, Y., Wang, C., Foster, L. J. \& Reiner, N. E. 2010a. An exosomebased secretion pathway is responsible for protein export from Leishmania and communication with macrophages. J. Cell Sci., 123:842-852.

Silverman, J. M., Clos, J., Horakova, E., Wang, A. Y., Wiesgigl, M., Kelly, I., Lynn, M. A., McMaster, W. R., Foster, L. J., Levings, M. K. \& Reiner, N. E. 2010b. Leishmania exosomes modulate innate and adaptive immune responses through effects on monocytes and dendritic cells. J. Immunol., 185:5011-5022.

Silverman, J. M. \& Reiner, N. E. 2011. Exosomes and other microvesicles in infection biology: organelles with unanticipated phenotypes. Cell. Microbiol., 13:1-9.

Tedesco, D. \& Haragsim, L. 2012. Cyclosporine: a review. J. Transplant., 2012:230386.

Wang, P. \& Heitman, J. 2005. The cyclophilins. Genome Biol., 6:226.

Wiesgigl, M. \& Clos, J. 2001. Heat shock protein 90 homeostasis controls stage differentiation in Leishmania donovani. Mol. Biol. Cell, 12:3307-3316.

Yau, W. L., Blisnick, T., Taly, J. F., Helmer-Citterich, M., SchieneFischer, C., Leclercq, O., Li, J., Schmidt-Arras, D., Morales, M. A., Notredame, C., Romo, D., Bastin, P. \& Spath, G. F. 2010. 
Cyclosporin A treatment of Leishmania donovani reveals stagespecific functions of cyclophilins in parasite proliferation and viability. PLoS Negl. Trop Dis., 4:e729.

Yau, W. L., Pescher, P., MacDonald, A., Hem, S., Zander, D., Retzlaff, S., Blisnick, T., Rotureau, B., Rosenqvist, H., Wiese, M., Bastin, P., Clos, J. \& Spath, G. F. 2014. The Leishmania donovani chaperone cyclophilin 40 is essential for intracellular infection independent of its stage-specific phosphorylation status. Mol. Microbiol., 93:80-97.

Zakai, H. A., Chance, M. L. \& Bates, P. A. 1998. In vitro stimulation of metacyclogenesis in Leishmania braziliensis, L. donovani, L. major and L. mexicana. Parasitology, 116(Pt 4):305-309.
Zilberstein, D. \& Shapira, M. 1994. The role of pH and temperature in the development of Leishmania parasites. Annu. Rev. Microbiol., 48:449-470.

1Present address: Department of Medical Biochemistry and Biophysics, Umeå University, 90187 Umeå, Sweden

${ }^{2}$ Present address: Stemcell Technologies, 500-1618 Station Street, Vancouver, British Columbia V6A 1B6, Canada ${ }^{3}$ Present address: Altona Diagnostics, Moerkenstr. 12, 22767 Hamburg, Germany 